\title{
Correction to: \\ The Protective Role of Dispositional Mindfulness Against the Perpetuation of Cyberbullying Victimization and Perpetration Among Adolescents, by Royuela-Colomer E, Calvete E, Gámez-Guadix M, and Orue I. Cyberpsychol Behav Soc Netw 2018;21(11):703-710. DOI: 10.1089/cyber.2017.0685
}

N THe November 2018 issue of Cyberpsychology, Behavior, and Social Networking (vol. 21, no. 11; 703-710), the article titled The Protective Role of Dispositional Mindfulness Against the Perpetuation of Cyberbullying Victimization and Perpetration Among Adolescents by Royuela-Colomer E, Calvete E, Gámez-Guadix M, and Orue I, requires a correction to Figure 2 on page 707.

"Low mindfulness" and "High mindfulness" were incorrectly identified in the Figure key.

Figure 2 should have appeared as:

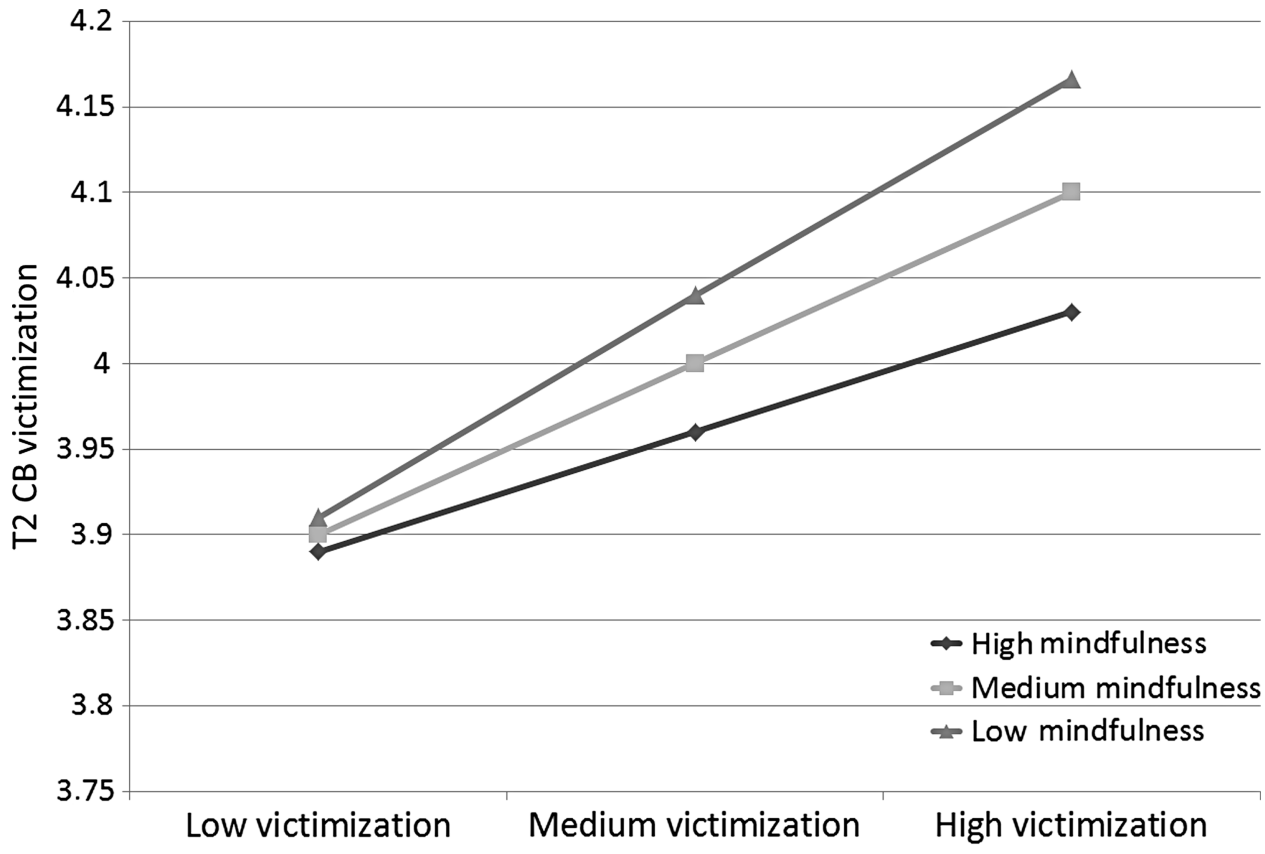

T1 CB victimization

The online version of the article has been corrected to reflect this change.

The authors apologize for the error. 